\title{
Unified Approach to Assess Engineering Performance of Fill Improved by Shallow to Deep Compaction Based Techniques Using Relative Density
}

\author{
Ahmed Alaaeldin'1, Emmanouil Spyropoulos², Anas Orabi ${ }^{3}$ \\ ${ }^{1}$ Keller Grundbau GmbH, Dammam, KSA \\ ${ }^{2}$ Saudi Aramco, Dhahran, KSA \\ ${ }^{3}$ Keller Grundbau GmbH, Dubai, United Arab Emirates \\ Email: ahmed.alaaeldin@keller.com, emmanouil.spyropoulos@aramco.com, anas.ourabi@keller.com
}

How to cite this paper: Alaaeldin, A., Spyropoulos, E. and Orabi, A. (2020) Unified Approach to Assess Engineering Performance of Fill Improved by Shallow to Deep Compaction Based Techniques Using Relative Density. Open Journal of Civil Engineering, 10, 239-249.

https://doi.org/10.4236/ojce.2020.103020

Received: August 6, 2020

Accepted: August 24, 2020

Published: August 27, 2020

Copyright ( 2020 by author(s) and Scientific Research Publishing Inc. This work is licensed under the Creative Commons Attribution International License (CC BY 4.0).

http://creativecommons.org/licenses/by/4.0/ (c) (i) Open Access

\begin{abstract}
The setting of pre assessment criteria for soil compaction is hardly determined, especially, in case of undecided structure locations. Different design guidelines recommend achieving a specific value of relative density for the compaction of fill placement works. Alternatives were discussed through the literature to predict the value of relative density based on soil field tests (e.g. cone and standard penetration tests). This paper presents the weakness of using the Over Consolidation Ratio (OCR) as guidance to assess the value of cone tip resistance using the soil relative density. The variation of OCR (from 1 to 10 ) has a significant effect on the $q_{c}$ value up to $110 \%$ when compared to the normally consolidated state. Then normally consolidated state can logically cover the compaction process with variation of $20 \%, 33 \%$, and $4 \%$ for relative density values $85 \%, 70 \%$, and $60 \%$, respectively. A unified approach is recommended to predict the compaction $q_{c}$-performance line using normally consolidated condition and sand relative density.
\end{abstract}

\section{Keywords}

Compaction, Relative Density, Performance Specifications

\section{Introduction}

In recent years, the field of construction has witnessed a great leap in building technologies with targeting a maximum cost saving. Nonetheless, the nature of 
the ground may obstacle this aim. Traditionally, for these cases, deep foundations (e.g. piles) are used to cope with the super-structure loads. Unfortunately, the using of piles leads to increase the cost of the project. Soil Improvement techniques are the most common solution utilized to achieve, for example, a proper soil bearing capacity with a target allowable settlement (i.e. the design criteria or the performance specifications) where the traditional over-excavation and replacement are not practicable for environmental, technical or economic reasons.

The design criteria for soil-treatment/fill-compaction may be unknown, especially, at the tender or site preparation stages in addition to undecided structures locations. However, the underneath soil/fill performance needs to be assessed prior to start the treatment process to determine the type and the depth of ground modifications.

This paper presents the weakness of using the Over Consolidation Ratio (OCR) as guidance to assess the value of cone tip resistance using the soil relative density. The variation of OCR (from 1 to 10) has a significant effect on the $q_{c}$ value up to $110 \%$ when compared to the normally consolidated state. A unified approach is recommended to predict the compaction $q_{c}$-performance line using normally consolidated condition and sand relative density.

\section{Background and Problem Statement}

Due to the difficulties in obtaining undisturbed samples of cohesionless soils, geotechnical engineers often rely on field tests to obtain in situ soil characteristics. A conventional analysis using Standard Penetration (SPT), Cone Penetration (CPT), or Pressure Meter (PMT) tests is suggested to check the minimum adequate criteria of a project [1]. The electronic CPT has emerged as one of the most popular tool for ground investigation due to its relatively lower cost, simplicity, continuous measurement with depth and excellent repeatability and accuracy. [2] and [3] suggested correlations between CPT results and soil characteristics such as unit weight $(\gamma)$, friction angle $(\varphi)$, relative density $\left(D_{r}\right)$, and elastic modulus (E).

However, the soil parameters to be utilized within the basic correlations of cone resistance and relative density is the analysis objective of this paper and whether Normally Consolidated (NC) or Over Consolidated (OC) concepts are applicable for the cases of densifying shallow soil formations. Scope is to define those parameters that are not over-conservative leading to excessive costs but also allows achieved soil compaction degrees that are safe for the subsequent top facilities construction. Therefore, an attempt is herein provided to show that through an integrated methodology the compaction $q_{c}$-performance line using normally consolidated condition and sand relative density is adequate to obtain the necessary densification amounts of the related soil formations without compromising the safety of the proposed structures upon such 
formations.

This paper presents the weakness of using the Over Consolidation Ratio (OCR) as a guidance to assess the value of cone tip resistance using the soil relative density. The variation of OCR (from 1 to 10) has a significant effect on the $q_{c}$ value up to $110 \%$ when compared to the normally consolidated state. A unified approach is recommended to predict the compaction $q_{c}$-performance line using normally consolidated condition and sand relative density.

\section{Relationships of Relative Density and Cone Resistance}

One of the most operational correlations is relating the measured cone tip resistance $\left(q_{c}\right)$ to the soil relative density as a factor to measure the compaction effectiveness. [4] and [5] performed calibration chambers tests, that was developed in 1969 [6], to appraise the $D_{r^{-}} q_{c}$ relationship. In a calibration chamber test with well-defined boundaries, a large cylindrical sand sample is deposited at a known soil properties (e.g. relative density) and consolidated to a desired stress state followed by a CPT (along the axis of the sample). Each test after completion provides one value of $q_{c}$ for a given value of $D_{r}$. The size and the boundaries conditions of the chamber are the most important parameters that affect the results as studied and listed by [7] and [8].

The value of the cone tip resistance $\left(q_{c}\right)$ can also be predicted based on the soil relative density $\left(D_{r}\right)$ as suggested by [9] in Equation (1) using $631 \mathrm{CPT}$ tests that collected from different calibration chamber test sources (i.e. [3] [10] [11] [12]). The utilized soil types were Ticino, Hokksund, Toyoura, Monterey, and Leighton Buzzard sands.

$$
q_{c}=C_{0} p_{a}\left(\frac{\sigma_{h}^{\prime}}{p_{a}}\right)^{C_{1}} D_{r}^{C_{2}}
$$

where, $C_{0}, C_{1}$, and $C_{2}$ are empirical constants that vary with the calibration chamber boundary conditions (see Table 1). The values of $C_{0}, C_{1}$, and $C_{2}$ equal to 360, 0.50, and 1.50 , respectively, had been recommended by the research authors to be generally utilized. $p_{a}$ is a reference or the atmospheric pressure, and $\sigma_{h}^{\prime}$ is the initial

Table 1 . The value of $C_{o}, C_{1}$, and $C_{2}$ according to chamber boundary conditions.

\begin{tabular}{ccccc}
\hline Constant & BC1 & BC2 & BC3 & BC4 \\
\hline $\begin{array}{c}\text { Lateral boundary } \\
\text { conditions }\end{array}$ & Constant stress & No displacement & No displacement & Constant stress \\
$\begin{array}{c}\text { Top and bottom } \\
\text { boundary conditions }\end{array}$ & Constant stress & No displacement & Constant stress & No displacement \\
$C_{o}$ & 350 & 320 & 370 & 320 \\
$C_{1}$ & 0.50 & 0.24 & 0.45 & 0.48 \\
$C_{2}$ & 1.51 & 1.10 & 1.51 & 1.29 \\
\hline
\end{tabular}


effective lateral stress $\left(\sigma_{h}^{\prime}=k_{0} \sigma_{v_{0}}^{\prime}\right.$ ) where $\sigma_{v_{0}}^{\prime}$ is the effective overburden pressure and $k_{0}$ is the at rest coefficient of lateral earth pressure $\left(k_{0}=(1-\sin \varphi) \mathrm{OCR}^{\sin \varphi}\right)$. OCR is the over consolidation ratio and $\varphi$ is the soil effective friction angle which can be calculated using soil relative density ( $\left.\varphi=28+0.15 D_{r},[13]\right)$.

[14] used the results of about 80 correlation calibration chamber tests on saturated Normally Consolidated (NC) sand, in addition to his work previously performed tests in 1976, to indicate the soil relative density from the cone tip resistance. The utilized samples were two artificial sands with opposite extreme crushabilities, two natural fine sands, and one natural and one artificial medium sands. Figure 1 presents the results obtained from the research.

[15] predicted the soil relative density for cohesionless soils based on calibration chamber tests on five different NC sands (Ticino, Ottawa, Edgar, Hokksund, and Hilton mines). The results produced the following relationship:

$$
D_{r}(\%)=68\left[\log \left(\frac{q_{c} / K_{q}}{\sqrt{p_{a} \sigma_{v_{0}}^{\prime}}}\right)-1\right]
$$

where, $K_{q}=1+\left(D_{r}-30\right) / 300$. It should be noted that an iteration process has to be applied to get the value of $D_{r}$.

[13] studied the normally consolidated and the over consolidated sand performance by using calibration chamber tests on Ticino and Hukksund sands. The following relation was obtained considering the calibration chamber boundary effects.

$$
D_{r}=\frac{1}{C_{2}} \ln \left(\frac{q_{c}}{C_{0} \sigma^{\prime C_{1}}}\right)
$$

The value of $\sigma^{\prime}=\sigma_{v_{0}}^{\prime}$ for normally consolidated sand and equal to $\sigma_{h}^{\prime}$ for over consolidated sand. The values of $C_{0}, C_{1}$, and $C_{2}$ had been recommended by the research authors for normally and over consolidated sandy soil as presented in Table 2. Nonetheless, the two series of coefficients for each soil case give very close results.

[16] finally suggested another formula to obtain the relative density from the cone tip resistance as shown in the following equation:

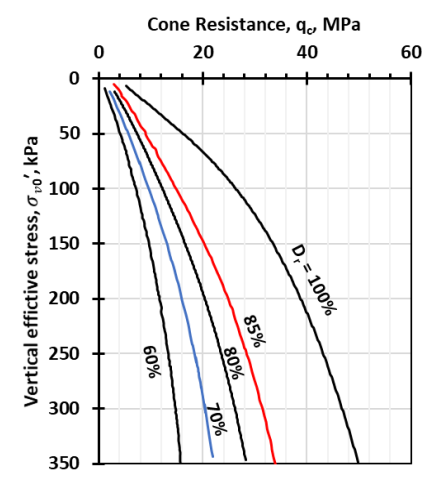

Figure 1. Cone tip resistance as a function of overburden pressure and soil relative density (after [14]). 
Table 2. The value of $C_{0}, C_{1}$, and $C_{2}$ according to stress history.

\begin{tabular}{cccccc}
\hline Soil Case & Sand type & $\sigma^{\prime}$ & $C_{0}$ & $C_{1}$ & $C_{2}$ \\
\hline \multirow{2}{*}{ NC } & Ticino & $\sigma_{v_{0}}^{\prime}$ & 157 & 0.55 & 2.41 \\
& Hukksand & & 86 & 0.53 & 3.29 \\
\multirow{2}{*}{ OC } & Ticino & \multirow{2}{*}{$\sigma_{h}^{\prime}$} & 220 & 0.53 & 2.64 \\
& Hukksand & & 170 & 0.54 & 3.01 \\
\hline
\end{tabular}

$$
D_{r}(\%)=\frac{1}{3.1} \ln \left[\frac{q_{c} / p_{a}}{17.68\left(\sigma_{v_{0}}^{\prime} / p_{a}\right)^{0.5}}\right]
$$

Based on the above-mentioned existing literature, the current assessment presents the weakness of using the Over Consolidation Ratio (OCR) as a guidance to assess the value of cone tip resistance using the soil relative density.

\section{Stress-Strain History Effect}

The over-consolidated (OC) soils, by means of dense state, can sustain larger loads when compared to the normally consolidated case. As such sands are generally identified as loose (behavior similar to NC clay) or dense (behavior similar to OC clay).The prediction of the soil relative density of the engineering performance of the soil is relatively unreliable. Although, the availability of correlations for estimating various parameters for soil (e.g. Elastic Modulus) depending on the NC and OC conditions.

By observing the behavior of NC and OC Sands, it is evident that the overconsolidated soil attains a higher shear strength comparing to the normally consolidated soil. However, both samples approach the same failure shear stress irrespective of the initial relative density, even though the OC soil exhibits more shear strength (dense soils dilate when sheared). This value is difficult to quantify in terms of relative density, so an effort to select a criteria that is more standardized and overall stronger is attempted.

As presented above, the value of $\sigma^{\prime}$ and the coefficients $C_{0}, C_{1}$, and $C_{2}$ shown in Equation (3) have been changed to be used in the same equation to account for over consolidation ratio [3]. [17] suggested the following ratio (Equation (5)) between the NC and OC $q_{c}$ values. Nevertheless, some other researchers proved that the $q_{c}$ value is slightly affected by the strain history of the sandy soil, on the other hand, the strain history considerably influences the sand stiffness ([3] [16] [18]).

$$
q_{c}^{O C}=q_{c}^{N C}\left[1+x\left(\mathrm{OCR}^{\beta}-1\right)\right]
$$

$$
\begin{aligned}
x & =0.75,([17]) \\
x & =0.50(\mathrm{OCR}=2) \text { to } 0.25(\mathrm{OCR}=15),([15]) \\
\beta & =0.42,([17])
\end{aligned}
$$


Table 3. The value of $Q_{f}$

\begin{tabular}{ccc}
\hline Soil Case & Sand State & $Q_{f}$ \\
\hline NC & Low, medium, high & 305 \\
& OCR $<3$ & 390 \\
OC & OCR $(3: 8)$ & 403 \\
& OCR $>8$ & 443 \\
\hline
\end{tabular}

$$
\beta=0.25+0.25 D_{r},([15])
$$

[19] carried out twelve Standard Penetration Tests (SPT) on normally (NC) and over consolidated $(\mathrm{OC})$ sands with $\mathrm{OCR}=3$. The results indicated that there is no effect of the soil stress history on SPT values which means that influence of the OCR value on the soil characteristics is negligible.

[20] provided a more coherent, straight-forward and simplistic approach (Equation (6)) to the estimation of Relative Density correlated from CPT $q_{c}$ values which accounted for the chamber boundary effects. The NC and OC tested sands were predominantly fine and medium sands in low, medium, and high compressibility states.

$$
D_{r}^{2}=\frac{1}{Q_{f} Q_{c} \mathrm{OCR}^{0.18}} \frac{q_{c} / p_{a}}{\left(\sigma_{v_{0}}^{\prime} / p_{a}\right)^{0.5}}
$$

where, $Q_{f}$ is a constant value which vary according to the soil state (Table 3). $Q_{c}$ is the compressibility factor which equals to $0.91,1.0$, and 1.09 for high, medium, and low compressibility, respectively.

\section{Over Consolidated Analysis}

The over consolidation ratio might be determined from the results of field tests (e.g. Cone Penetration Tests) However, it is very difficult to estimate the value of OCR from the energy produced by the top-bottom compaction (Dynamic and Rapid Impact Compactions).

Figure 2(a) shows the influence of the type of the sand, utilized by [3], on the values of cone resistance. As glanced from the graph, a negligible difference can be observed between the results of Ticino and Hukksand sands for OCR $=3.0$. A significant effect is witnessed by changing the OCR value from 1.0 to 3.0 for [3] and [20], see Figure 4(b) and Figure 4(c). Table 4 presents the sand properties used for this study.

Figure 3 presents the $q_{c}$-profile predicted based on $85 \%$ sand relative density and OCR $=3.0$ up to $4.0 \mathrm{~m}$ depth using the different approaches presented above. The value of $q_{c}$ obtained from [20] increases by $24 \%, 44 \%, 59 \%$, and $73 \%$ when compared to that attained using [3] [9] [14] [15] equations, respectively. While, a maximum variation of $10 \%$ to $20 \%$ is observed between the correlations of [3] [9] [14]. However, a unified approach cannot be prepared using OCR $=3$ as this value depends on the stress-strain history state which cannot easily estimated as a project criteria from compaction process. 


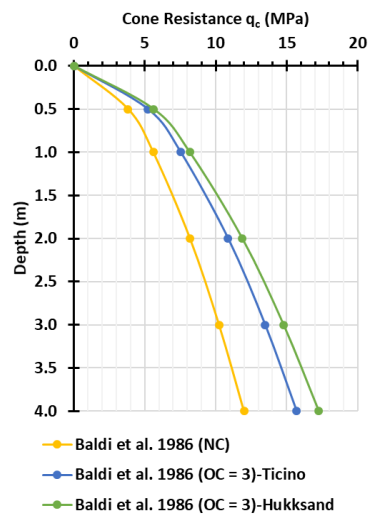

(a)

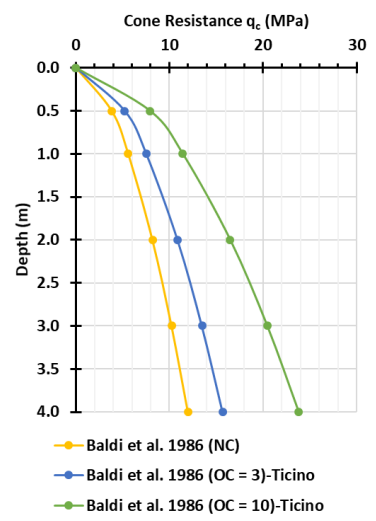

(b)

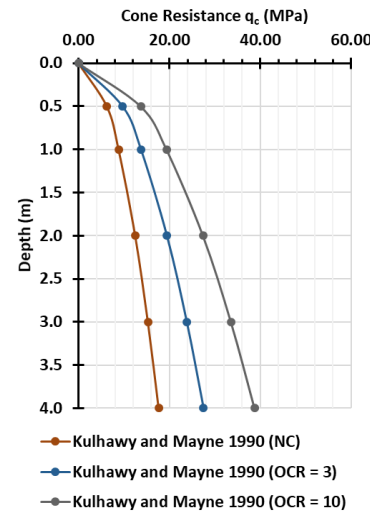

(c)

Figure 2. Effect of over consolidation ratio on the cone tip resistance performance line. (a) Effect of sand type on the value of $q_{c}$; (b) Variation of OCR from [3]; (c) Variation of OCR from [20].

Table 4. Sand properties used in the analysis.

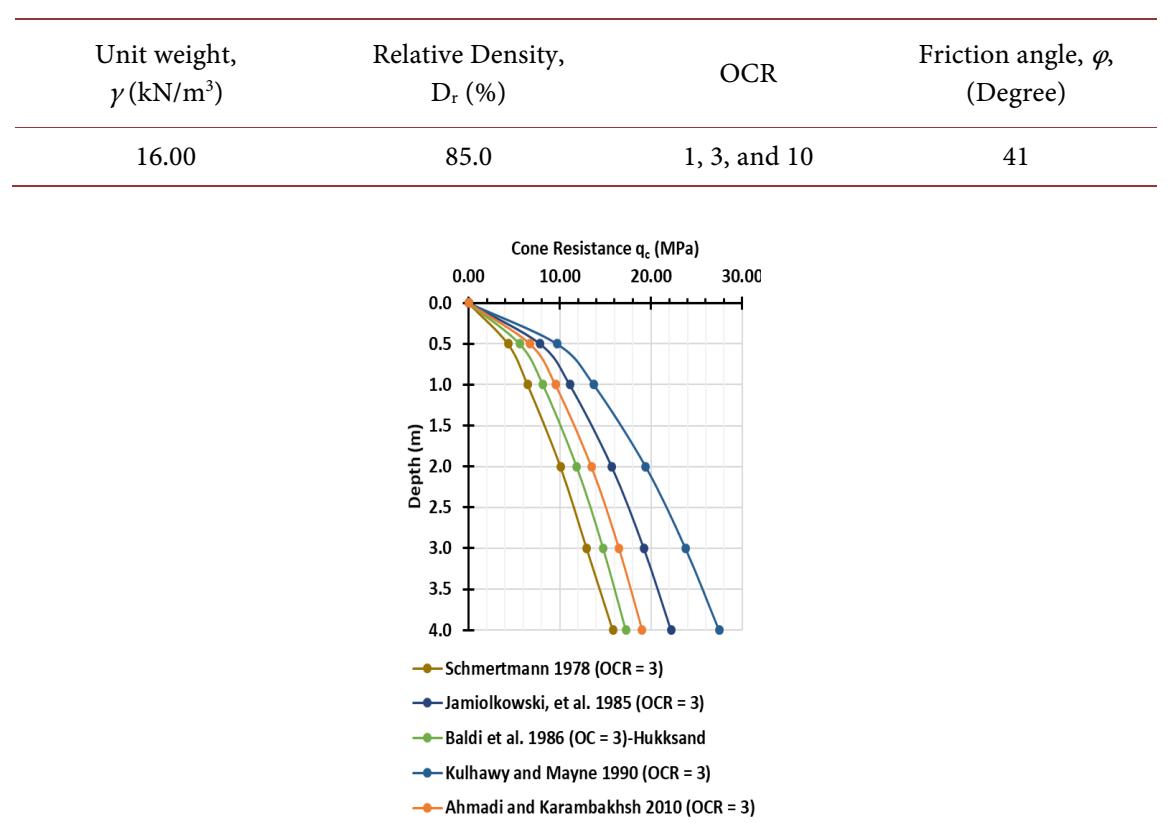

Figure 3. $q_{c}$ profile based on over consolidation ratio $(\mathrm{OCR}=3)$.

\section{Normally Consolidated Analysis}

For normally consolidated analysis, where the preconsolidation pressure equals to the existing overburden pressure, the approaches presented above are utilized and the results are presented in Figure 4. As indicated from Figure 4(a) $\left(D_{r}=\right.$ 85\%), [15] and [20] almost have the same predicted $q_{c}$-value which soar by $40 \%$ from the nearest results. Whereas, the other three correlations have a maximum difference of 20\%. On the other hand, for $D_{r}=70 \%$ (see Figure 4 (b)), the results of [14] have the lowest values by $33 \%$ less than the nearest approach (i.e. [3]). Nonetheless, in this case a unified approach may be followed as the value of 


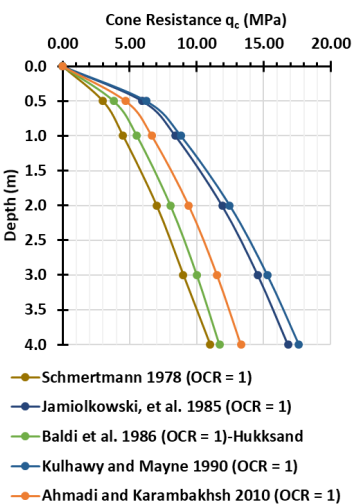

(a)

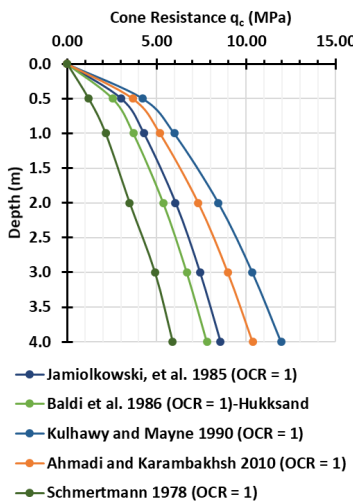

(b)

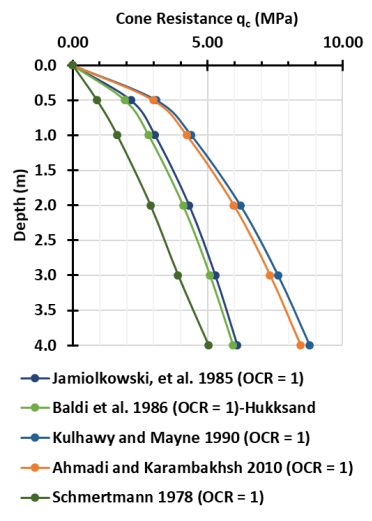

(c)

Figure 4. $q_{c}$ profile for Normally consolidated sands. (a) $D_{r}=85 \%$; (b) $D_{r}=70 \%$; (c) $D_{r}=$ $60 \%$.

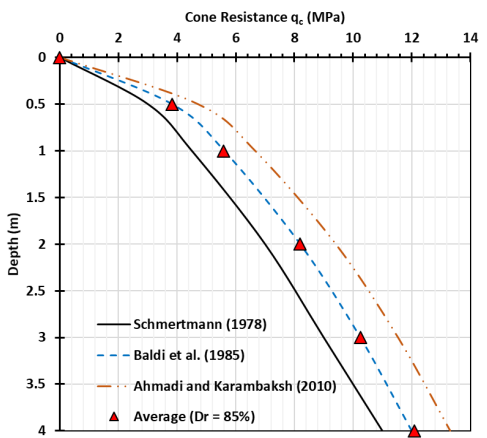

(a)

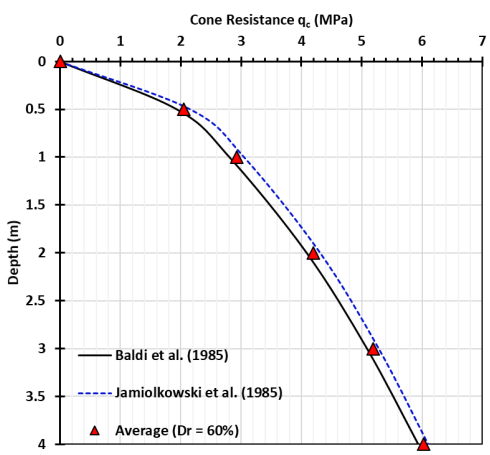

(c)

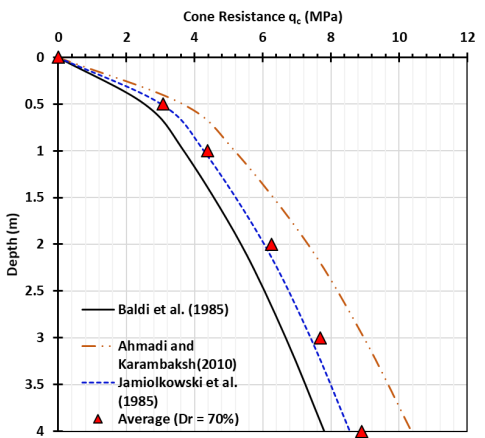

(b)

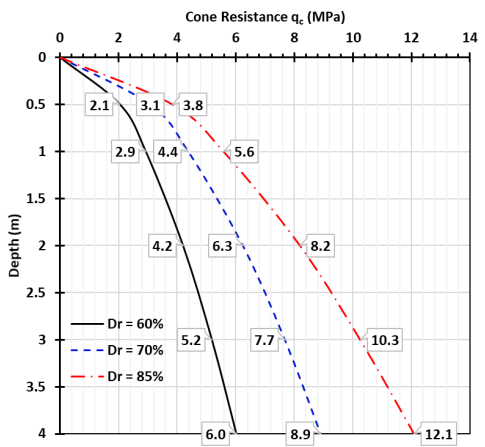

(d)

Figure 5. Recommended $\mathrm{q}_{\mathrm{c}}$ performance line based on sand relative density. (a) $q_{c}$-profile for $D_{r}=85 \%$; (b) $q_{c}$-profile for $D_{r}=70 \%$; (c) $\mathrm{q}_{\mathrm{c}}$-profile for $D_{r}=60 \%$; (d) Average $q_{c}$-profiles.

OCR = 1 for normally consolidated sandy soil.

As a result, the formulas suggested by [3] [9] [14] can be merged or used separately to assess the $q_{c}$ values along the entire depth for $D_{r}=85 \%$. While, all of the correlations except [14] and [20] may be applied for $D_{r}=70 \%$. Moreover, only [3] and [15] could be utilized as these two formulas represent the average between other suggestions. 


\section{Performance Criteria}

Based on the above mentioned points and by assuming that the value of OCR will not be affected rapidly after the treatment process because of hammering which breaks the bonding between soil particles and, also, the stress-history effect need some time until the soil be remolded and recemented. Therefore, for non-preloaded areas, the normally consolidated criteria may be applied to assess a CPT cone resistance performance line, especially, if there is no decided other criteria such as the bearing capacity and settlement.

By determining the anticipated value of relative density, Figure 5 can be used to predict the performance line of the cone tip resistance for sandy soils up to 4.0 $m$ depth which is the usual soil thickness that the Rapid Impact Compaction soil improvement method can mitigate on a single run from the top surface.

\section{Conclusions}

Due to the lack of information provided before finalizing the foundation design of a project, a criteria for performance achievement needs to be placed. By spotting the recommendation of different specifications to achieve sufficient relative compaction/density, this paper studied the different alternatives presented on the literature to unify the criteria of using relative density to assess a compaction performance line.

However, the soil parameters to be utilized within the basic correlations of cone resistance and relative density and whether Normally Consolidated (NC) or Over Consolidated (OC) concepts are applicable for the cases of densifying shallow soil formations was examined.

Having completed the analysis of the existing literature, it was concluded that the Over Consolidation Ratio (OCR) cannot be utilized to suggest a guidance to assess the value of cone tip resistance using the soil relative density. The variation of OCR (from 1 to 10 ) has a significant effect on the $q_{c}$ value up to $110 \%$ when compared to the normally consolidated state. Therefore, an attempt was provided to show that through an integrated methodology the compaction $q_{c}$-performance line using normally consolidated condition and sand relative density is adequate to obtain the necessary densification amounts of the related soil formations without compromising the safety of the proposed structures upon such formations. It was concluded that the normally consolidated state can logically cover the compaction process with variation of $20 \%, 33 \%$, and $4 \%$ for relative density values $85 \%, 70 \%$, and $60 \%$, respectively.

\section{Conflicts of Interest}

The authors declare no conflicts of interest regarding the publication of this paper.

\section{References}

[1] FHWA (2017) Ground Modification Methods. Federal Highway Administration (FHWA), Washington DC. 
[2] Robertson, P.K. and Campanella, R.G. (1983) Interpretation of Cone Penetration Tests: Sands and Clays. Canadian Geotechnical Journal, 20, 719-745. https://doi.org/10.1139/t83-079

[3] Baldi, G., Bellotti, R., Ghionna, V., Jamiolkowski, M. and Pasqualini, E. (1986) Interpretations of CPT's and CPTU's, 2nd Part: Drained Penetration of Sands. 4th International Conference on Field Instrumentation and in-Situ Measurements, Singapore, November 1986, 143-156.

[4] Olsen, R.S. (1994) Normalization and Prediction of Geotechnical Properties Using the Cone Penetrometer Test (CPT). US Army Engineer Waterways Experiment Station.

[5] Gupta, R., Rodrigo, S., Mitchell, J. and Jamiolkowski, M. (2001) Calibration Chamber Size Effects on Penetration Resistance in Sand. Journal of Geotechnical and Geoenvironmental Engineering, 127, 628.

[6] Holden, J.C. (1971) Research on Performance of Soil Penetrometers. Internal Report, Country Roads Board of Victoria, Victoria.

[7] Ghionna, V.N. and Jamliolkowski, H. (1991) A Critical Appraisal of Calibration Chamber Testing of Sands. Proceedings of the 1 st International Symposium on Calibration Chamber Testing, Potsdam, 28-29 June 1991, 13-39.

[8] Salgado, R., Mitchel, J.K. and Jamiolkowski, M. (1998) Calibration Chamber Size Effects on Penetration Resistance in Sand. Journal of Geotechnical and Geoenvironmental Engineering, 124, 878-888. https://doi.org/10.1061/(ASCE)1090-0241(1998)124:9(878)

[9] Ahmadi, M.M. and Karambakhsh, P. (2010) Ko Determination of Sand Using CPT Calibration Chamber. 2nd International Symposium on Cone Penetration Testing, Huntington Beach, May 2010, 1-8.

[10] Salgado, R. (1993) Analysis of Penetration Resistance in Sand. PhD Thesis, University of California, Berkeley.

[11] Lunne, T., Robertson, P.K. and Powell, J.M. (1997) Cone Penetration Testing in Geotechnical Practice. Blackie Academic \& Professional, London.

[12] Houlsby, G.T. and Hitchman, R.C. (1988) Calibration Tests of Cone Penetrometers in Sand. Géotechnique, 38, 39-44. https://doi.org/10.1680/geot.1988.38.1.39

[13] Meyerhof, G.G. (1959) Compaction of Sands and Bearing Capacity of Piles. Transactions of the American Society of Civil Engineers, 126, 1292-1322.

[14] Schmertmann, J.H. (1978) Guidelines for Cone Penetration Test, Performance and Design. Report FHWA-TS-78-209, Federal Highway Administration, Washington DC.

[15] Jamiolkowski, M., Baldi, G., Belloti, R., Ghionna, V. and Pasqualini, E. (1985) Penetration Resistance and Liquefaction of Sands. Proceedings of the 11 th International Conference on Soil Mechanics and Foundation Engineering, 57-153.

[16] Jamiolkowski, M., Lo Presti, D.C.F. and Manassero, M. (2001) Evaluation of Relative Density and Shear Strength of Sands from CPT and DMT. Soil Behavior and Soft Ground Construction: Geotechnical Special Publication, 11, 201-238.

[17] Schmertmann, J.H. (1975) Measurement of In-Situ Shear Strength. Proceedings of ASCE Spec. Conference on In-Situ Measurement of Soil Properties, Raleigh, NC, Vol. 2, 57-138.

[18] Marchetti, S. (1982) Detection of Liquefiable Sand Layers by Means of Quasi-Static 
Penetration Sand Layers by Means of Quasi-Static Penetration. ESPOT II, Amsterdam.

[19] Bieganousky, W.A. and Marcuson, W.F. (1976) Liquefaction Potential of Dams and Foundations, Report No. 1, Laboratory Standard Penetration Tests on Reid Bedford Model and Ottawa Sands. Research Reports-76-2, W.E.S Vicksburg, Miss.

[20] Kulhawy, F.H. and Mayne, P.H. (1990) Manual on Estimating Soil Properties for Foundation Design. Report EL-6800, Electric Power Research Institute, Washington DC. 